\title{
Psychological/neuropsychological testing: When to refer for reexamination
}

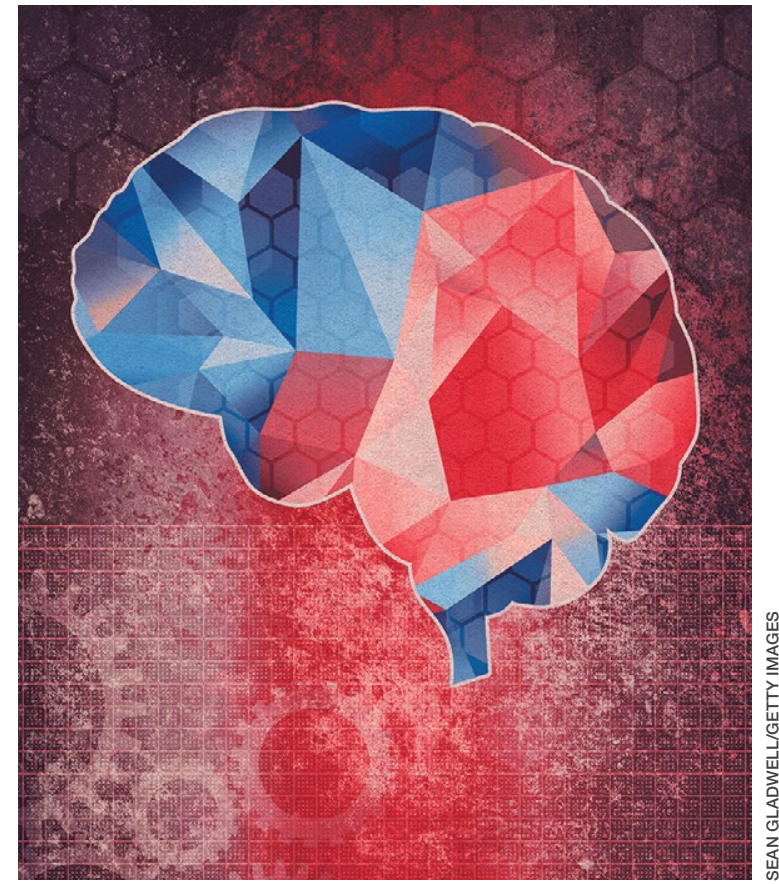

\section{Jerrold Pollak, PhD, ABPP, ABN}

Clinical and Neuropsychologist and Emergency Services Clinician Seacoast Mental Health Center

Portsmouth, New Hampshire

\section{Understanding when retesting is most useful can help clarify diagnosis and treatment}

$\mathrm{T}$ he evolution of illness prevention, diagnosis, and treatment has involved an increased appreciation for the clinical utility of longitudinal assessment. This has included the implementation of screening evaluations for high base rate medical conditions, such as cancer, that involve considerable morbidity and mortality.

Unfortunately, the mental health professions have been slow to embrace this approach. Baseline assessment with psychological/neuropsychological screening tests and more comprehensive test batteries to clarify diagnostic status and facilitate treatment planning is far more the exception than the rule in mental health care. This seems to be the case despite the strong evidence supporting this practice as well as multiple surveys indicating that psychiatrists and other physicians report a high level of satisfaction with the findings and recommendations of psychological/neuropsychological test reports. ${ }^{1-3}$

There is a substantial literature that reviews the relative indications and contraindications for initial psychological/neuropsychological test evaluations. ${ }^{4-7}$ However, there is a paucity of clinical and evidence-based information regarding criteria for follow-up assessment. Moreover, there are no consensus guidelines to inform decision-making regarding this issue.

In general, good clinical practice for baseline assessment and reexamination should include administration of both psychological and neuropsychological tests. Based on clinical experience, this article addresses the relative indications and contraindications for psychological/neuropsychological

\section{Disclosure}

The author reports no financial relationships with any companies whose products are mentioned in this article, or with manufacturers of competing products.

doi: 10.12788/cp.0157 
test reassessment of adults seen in psychiatric care. It also outlines suggested time frames for such reevaluations, based on the patient's clinical status and circumstances.

\section{Why are patients not referred for reassessment more often?}

There are several reasons patients are not referred for follow-up testing, beginning with the failure, at times, of the psychologist to state in the recommendations section of the test report whether a reassessment is indicated, under what circumstances, and within what time frame. Empirical data is lacking, but predicated on clinical experience, even when a strong and unequivocal recommendation is made for reassessment, only a very small percentage of patients are seen for follow-up evaluation.

There are numerous reasons why this occurs. The patient and/or the psychiatrist may overlook or forget about the recommendation for reassessment, particularly if it was embedded in a lengthy list of recommendations and the suggested time frame for the reassessment was several years away. The patient and the psychiatrist may decide against going forward with a reexamination, for a variety of substantive reasons. The patient might decline, against medical advice, to be retested. The patient may fail to make or keep an appointment for the follow-up reexamination. The patient might leave treatment and become lost to follow-up. The patient might not be able to find an appropriate psychologist. The insurance company may decline to authorize follow-up testing. ${ }^{8}$

\section{Indications for reevaluation}

Follow-up testing generally is indicated in the following circumstances:

Patients who are likely to soon improve or worsen. Reassessment is indicated when, based on the initial evaluation, the patient has been identified as having a neuropsychiatric disorder that is likely to improve or worsen over the next year or 2 due to the natural trajectory of the condition and the degree to which it may respond to treatment.
Patients who are likely to improve include those with mental status changes referable to $\geq 1$ medical and/or neuropsychiatric factors that are considered at least partially treatable and reversible. Patients who fall within this category include those who have mild to moderately severe head trauma or stroke, have a suspected or known medication- or substance-induced altered mental status, appear to have depression-related cognitive difficulties, or have an initial or recurrent episode of idiopathic psychosis.

Patients whose conditions can be expected to worsen over time include those with a mild neurocognitive disorder or major neurocognitive disorder of mild severity that is considered referable to a progressive neurodegenerative illness such as Alzheimer's disease based on family and personal history, their psychometric test profile, and other factors, including findings from positron emission tomography scanning.

Older patients who were referred primarily due to a strong family history of major neurocognitive disorder but with no clear-cut concerning findings on baseline testing warrant reevaluation in the event of the emergence of significant cognitive and/or psychiatric symptoms and/or a functional decline since the baseline examination.

Patients who have been seen for initial test evaluations prior to interventions such as neurosurgery (including psychosurgery), electroconvulsive therapy (ECT), transcranial magnetic stimulation, cognitive rehabilitation, etc.

Patients undergoing a substantial transition. Reevaluation is appropriate for a broad range of patients experiencing difficulties when undergoing a significant lifestyle transition or change in level of care. This includes patients considering a return to school or work after a prolonged absence due to neuropsychiatric illness, or for whom there are questions regarding the need for a change in their level of everyday care. The latter includes patients who are returning to home care from assisted living, or transferring from home-based services to assisted living or a skilled nursing facility.

What about patients with psychiatric disorders? A "grey area" pertains to reassessment of patients with neuropsychiatric disorders

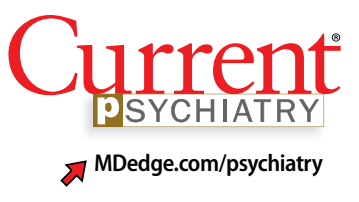

Clinical Point

Reassessment is indicated for patients with a neuropsychiatric illness that is likely to soon improve or worsen 


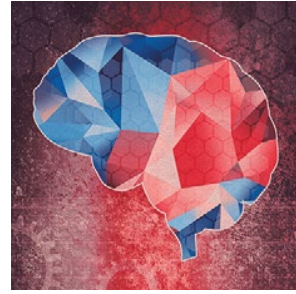

Neuropsychological reassessment

\section{Clinical Point}

\section{Consider}

reevaluation for

patients with any

factors that suggest

a progressive, deteriorative course of illness

\section{Table}

\section{Psychological/neuropsychological reexamination: Indications and contraindications}

Indications

- Patients who are likely to soon improve or worsen

- Older patients who were referred primarily due to a strong family history of major neurocognitive disorder but with no clear-cut concerning findings on baseline testing

- Patients who have been seen for initial test evaluations prior to interventions such as neurosurgery or ECT

- Patients undergoing a significant lifestyle transition or change in level of care

\section{Contraindications}

- Patients with advanced major neurocognitive disorder

- Patients with transient episodes of poor functioning

- Patients who have not improved or worsened over an extended time, and a protracted change is not anticipated

A 'grey area' pertains to reassessment of patients with neuropsychiatric disorders such as schizophrenia, bipolar disorder, MDD, and OCD. Consider reevaluation for patients with a family and/ or personal history of cognitive/neuropsychological impairment, structural brain abnormalities on neuroimaging, a concerning cognitive/neuropsychological profile, or any other factors that raise the index of suspicion for a possible progressive deteriorative course of illness

ECT: electroconvulsive therapy; MDD: major depressive disorder; OCD: obsessive-compulsive disorder

Source: References 9-14

such as schizophrenia and related psychotic disorders, bipolar disorder, major depressive disorder, and obsessive-compulsive disorder. Patients with these conditions often have high rates of cognitive/neuropsychological impairment on baseline testing, even when they appear to be improving from a psychiatric perspective, are reasonably stable, and may even be in remission. ${ }^{9-12}$

These deficits are frequently a mix of preillness, prodromal, and early-stage illnessrelated neurocognitive difficulties that, for the most part, remain stable over time. That said, there is emerging evidence of worsening cognitive change over time following a first episode of psychosis for some patients with schizophrenia. ${ }^{13}$

In general, reevaluation should be considered for patients with a family and/or personal history of cognitive/neuropsychological impairment, structural brain abnormalities on neuroimaging, a concerning cognitive/neuropsychological profile, or any other factors that raise the index of suspicion for a possible progressive deteriorative course of illness. ${ }^{13,14}$

Patients with personality disorders who have had a baseline psychometric evaluation do not clearly warrant reassessment unless they develop medical and/or psychosocial difficulties that are often linked to problematic personality traits/patterns and that result in significant and persistent mental status changes. For example, reassessment might be indicated for a patient with borderline personality disorder who has new-onset or worsening cognitive and/or psychiatric complaints/symptoms after sustaining a head injury while intoxicated and embroiled in a domestic conflict triggered by anger and fears related to abandonment and separation.

Reevaluation also should be considered when a patient with a personality disorder has had a baseline assessment and subsequently completes an intensive, long-term treatment program that is likely to improve their clinical status. In this context, retesting may help document these gains. Examples of such programs/services include residential psychiatric and/or substance abuse care, object relational/psychodynamically-based psychotherapy, an extended course of dialectical behavioral therapy, or a related coping skills/distress tolerance psychotherapy.

\section{Contraindications for reassessment}

Retesting generally is not indicated in the following circumstances:

Patients with advanced major neurocognitive disorder. Reassessment is not indicated for such patients when there are no new questions regarding diagnosis, prognosis, 
level of care, and/or related disposition issues.

Patients with transient episodes of poor functioning. For the most part, reassessment is not helpful for patients with well-established diagnoses and treatment plans who, based on their history, experience time-limited, recurrent episodes of poor functioning and then reliably return to their baseline with ongoing psychiatric care. This includes patients with borderline personality disorder and other personality difficulties with histories of transient decompensation in response to psychosocial and psychodynamic triggers.

Patients who do not improve or worsen over time. Reassessment is not indicated when there has been no clear, sustained improvement or worsening of a patient's clinical status over an extended time, and a protracted change is not anticipated. In this situation, reassessment is unlikely to yield clinically useful information beyond what is already known or meaningfully impact case formulation and treatment planning.

The Table T-14 $^{9}$ (page 30) summarizes the relative indications and contraindications for psychological/neuropsychological reexamination.

\section{Time frames for reassessment}

Time frames for retesting vary considerably depending on factors such as diagnostic status, longitudinal course, treatment parameters, and recent/current life circumstances.

While empirical data is lacking regarding this matter, based on clinical experience, reevaluation in 18 to 24 months is generally appropriate for patients with

\section{Related Resources}

- Thom R, Farrell HM. Neuroimaging in psychiatry: potentials and pitfalls. Current Psychiatry. 2019;18(12):27-28;33-34.

- Papakostas Gl. Cognitive symptoms in patients with major depressive disorder and their implications for clinical practice. J Clin Psychiatry. 2014;75(1):8-14.

- American Board of Professional Neuropsychology. https:// abn-board.com

neuropsychiatric conditions who are likely to gradually improve or slowly worsen over this time. Still, reexamination can be sooner (within 12 to 18 months) for patients who have experienced a more rapid and steep negative change in clinical status than initially anticipated.

For most patients with major mental illness, reexamination in 3 to 5 years is probably a reasonable time interval, barring a poorly understood and clinically significant negative change in functioning that warrants a shorter time frame. This suggested time frame would allow for sufficient time to better gauge improvement, stability, or deterioration in functioning and whether the reason(s) for referral have evolved. On the other hand, this time interval is somewhat arbitrary given the lack of empirical data. Therefore, on a case-by-case basis, it would be helpful for psychiatrists to consult with their patients and preferably with the psychologist who completed the baseline evaluation to determine a reasonable interval between assessments.

For patients who have undergone longterm/intensive treatment, reassessment in 3 or 6 months to as long as 1 year after the patient completes the program should be considered. Patients who undergo medical continued on page 37

\section{Bottom Line}

Only a very small percentage of patients referred for follow-up psychological/ neuropsychological test reevaluation actually undergo reexamination. Such retesting may be most helpful for certain patient populations, such as those who are likely to soon improve or worsen, were referred based on a family history of major cognitive disorder but have no concerning findings on baseline testing, or are undergoing a substantial life transition.

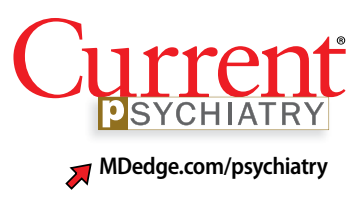

Clinical Point

Reassessment is not helpful for patients who experience time-limited, recurrent episodes of poor functioning and then return to baseline 
interventions such as neurosurgery or ECT - which can be associated with shortterm, at least partially reversible negative effects on mental status-reassessment usually is most helpful when initiated as one or more screening level examinations for several weeks, followed by a comprehensive psychometric reassessment at the 3- to 6-month mark.

\section{Suggestions for future research}

Additional research is needed to ascertain the attitudes and opinions of psychiatrists and other physicians who use psychometric test data regarding how psychologists can most effectively communicate a recommendation for reassessment in their reports and clarify the ways psychiatrists can productively address this issue with their patients. Survey research of this kind should include questions about the frequency with which psychiatrists formally refer patients for retesting, and estimates of the rate of follow-through.

It also would be desirable to investigate factors that may facilitate follow-through with recommendations for reassessment, or, conversely, identify reasons that psychiatrists and their patients may decide to forgo reassessment. It would be important to try to obtain information regarding the optimum time frames for such reevaluation, depending on the patient's circumstances and other variables. Evidence-based data pertaining to these issues would contribute to the development of consensus guidelines and a standard of care for psychological/ neuropsychological test reevaluation.

\section{References}

1. Schroeder RW, Martin PK, Walling A. Neuropsychological evaluations in adults. Am Fam Physician. 2019;99(2): 101-198.

2. Bucher MA, Suzuki T, Samuel DB. A meta-analytic review of personality traits and their associations with mental health treatment outcomes. Clin Psychol Rev. 2019;70:51-63.

3. Pollak J. Feedback to the psychodiagnostician: a challenge for assessment psychologists in independent practice. Independent Practitioner: The community of psychologists in independent practice. 2020;40,6-9.

4. Pollak J. To test or not to test: considerations before going forward with psychometric testing. The Clinical Practitioner. 2011;6:5-10.

5. Schwarz L, Roskos PT, Grossberg GT. Answers to 7 questions about using neuropsychological testing in your practice. Current Psychiatry. 2014;13(3):33-39.

6. Zucchella C, Federico A, Martini A, et al. Neuropsychological testing. Pract Neurol. 2018;18(3):227-237.

7. Moller MD, Parmenter BA, Lane DW. Neuropsychological testing: a useful but underutilized resource. Current Psychiatry. 2019;18(11):40-46,51

8. Pollak J. Psychodiagnostic testing services: the elusive quest for clinicians. Clinical Psychiatry News. Published October 18, 2019. Accessed July 8, 2021. https://www. mdedge.com/psychiatry/article/210439/schizophreniaother-psychotic-disorders / psychodiagnostic-testingservices

9. Mesholam-Gately RI, Giuliano AJ, Goff KP, et al. Neurocognition in first episode schizophrenia: a metaanalytic review. Neuropsychology. 2009;23(3):315-336.

10. Lam RW, Kennedy SH, McIntyre RS et al. Cognitive dysfunction in major depressive disorder: effects on psychosocial functioning and implications for treatment. Can J Psychiatry. 2014;59(12):649-654.

11. Szmulewicz AG, Samamé C, Martino DJ, et al. An updated review on the neuropsychological profile of subjects with bipolar disorder. Arch Clin Psychiatry. 2015;42(5):139-146.

12. Shin NY, Lee TY, Kim, E, et al. Cognitive functioning in obsessive-compulsive disorder: a meta-analysis. Psychol Med. 2013;44(6):1121-1130.

13. Zanelli J, Mollon J, Sandin S, et al. Cognitive change in schizophrenia and other psychoses in the decade following the first episode. Am J Psychiatry. 2019;176(10):811-819.

14. Mitleman SA, Buchsbaum MS. Very poor outcome schizophrenia: clinical and neuroimaging aspects. Int Rev Psychiatry. 2007;19(4):345-357.

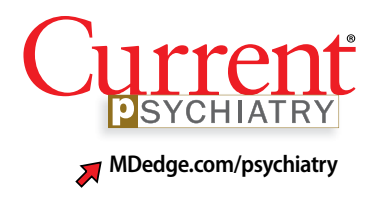

Clinical Point

For most patients with major mental illness, reexamination in 3 to 5 years is probably a reasonable time interval 